This item was submitted to Loughborough's Research Repository by the author.

Items in Figshare are protected by copyright, with all rights reserved, unless otherwise indicated.

\title{
Capturing localised extinction in Sandia Flame F with LES-CMC
}

PLEASE CITE THE PUBLISHED VERSION

http://dx.doi.org/10.1016/j.proci.2010.06.065

\section{PUBLISHER}

(C) The Combustion Institute. Published by Elsevier Inc.

\section{VERSION}

AM (Accepted Manuscript)

LICENCE

CC BY-NC-ND 4.0

REPOSITORY RECORD

Garmory, Andrew, and E. Mastorakos. 2012. "Capturing Localised Extinction in Sandia Flame F with LESCMC”. figshare. https://hdl.handle.net/2134/11116. 
This item was submitted to Loughborough's Institutional Repository (https://dspace.lboro.ac.uk/) by the author and is made available under the following Creative Commons Licence conditions.

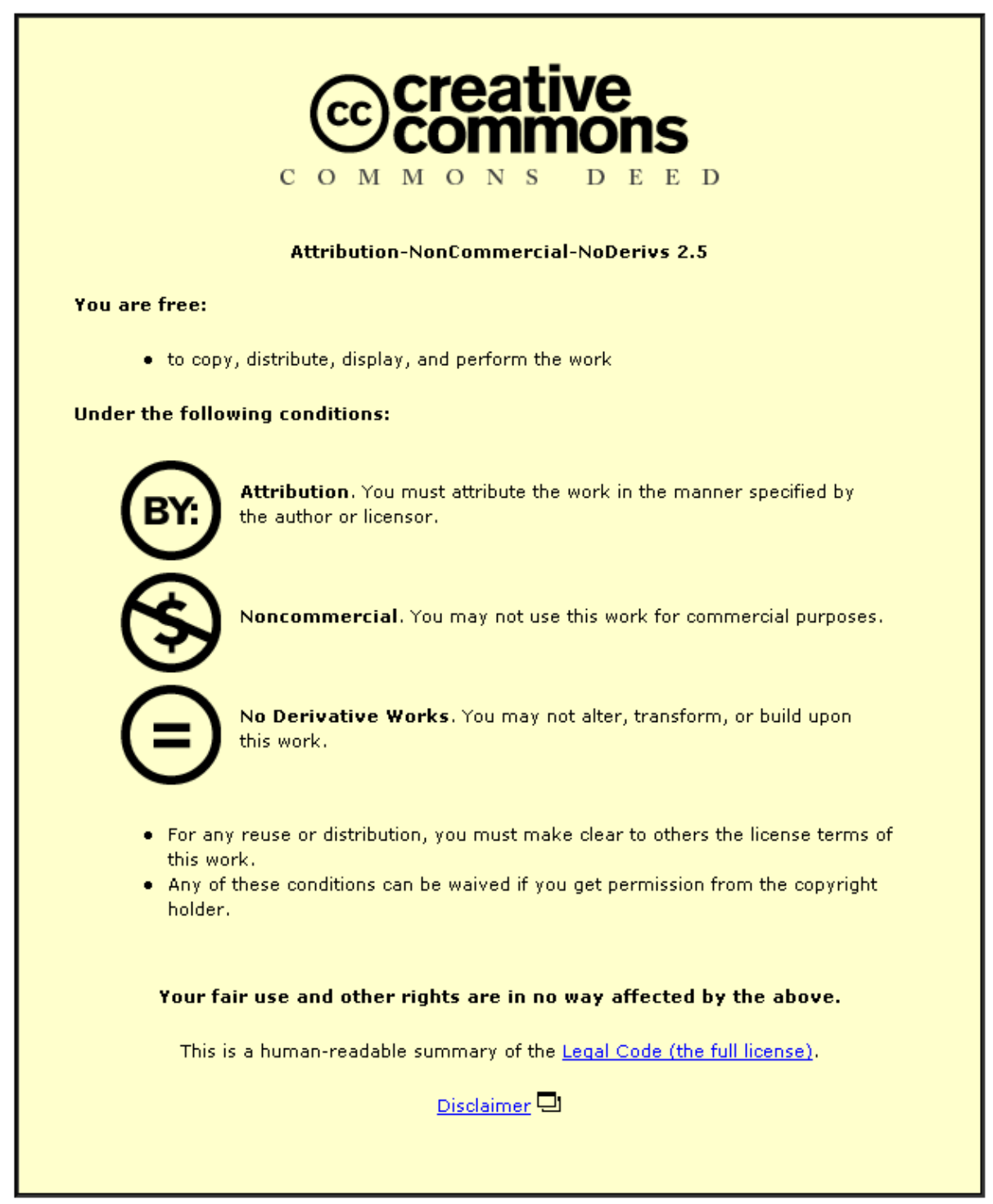

For the full text of this licence, please go to: http://creativecommons.org/licenses/by-nc-nd/2.5/ 


\title{
Capturing Localised Extinction in Sandia Flame F with LES-CMC
}

\author{
A. Garmory ${ }^{\mathrm{a}, *}$, E. Mastorakos ${ }^{\mathrm{a}}$ \\ ${ }^{a}$ Hopkinson Laboratory, Department of Engineering, University of Cambridge, \\ Cambridge CB2 1PZ, United Kingdom
}

\begin{abstract}
A Large Eddy Simulation (LES) using the Conditional Moment Closure (CMC) as a sub-grid turbulence-chemistry model has been applied to piloted jet diffusion flames (Sandia D \& F). A 3D CMC grid was used which allowed different CMC boundary conditions to be applied in the jet and pilot streams. The code was found to give very good agreement with experiment in the low extinction case of Flame D. For Flame F transient extinction and reignition events were observed with LES-CMC which lead to reductions in averaged unconditional and conditional temperature consistent with experimental observations. Further analysis revealed that the CMC extinction/ignition events were the result of a combination of high conditional scalar dissipation rate and transport in the CMC grid.
\end{abstract}

Keywords:

LES, CMC, extinction, re-ignition, non-premixed

\section{Introduction}

Large Eddy Simulation (LES) is being used increasingly widely to predict the behaviour of turbulent combustion [1]. In LES the large scales in the flow are solved directly while the smaller scale effects must be modelled. Chemical reaction is a phenomenon that occurs at the smallest scales in the flow and hence a turbulent combustion model must be used with the LES. These include steady [2] and unsteady [3] flamelet models, the flamelet/progress

\footnotetext{
*Corresponding author

Email address: ag310@cam.ac.uk (A. Garmory )
} 
varible (FPV) model [4] and the Stochastic Fields or Eulerian Monte Carlo method [5].

The Sandia piloted jet flames [6] have been the subject of several numerical studies due to the detailed experimental data for both scalar and velocity fields and also to the challenge of correctly predicting the localised extinction behaviour of the faster flames. Previous studies using transported PDF methods in RANS have produced good agreement with experiment $[7,8]$ for flame $\mathrm{F}$ and also revealed the sensitivity of this flame $\mathrm{F}$ to the chosen chemical mechanism [9]. These studies were useful in determining the parameters needed in RANS-PDF modelling to give accurate results, a process that is now being undertaken for LES studies. The presence of localised extinction in flame $\mathrm{E}$ has successfully been predicted in [10] using the FPV model.

Here, we use Conditional Moment Closure (CMC) which has previously been used in an LES context for Sandia D [11], bluff-body steady flames [12], autoigniting jets [13] and for spark ignition problems [14]. In this method the assumption is made that while reactive scalar values might fluctuate strongly, their fluctuations about a value conditionally averaged on the value of some conserved scalar will be small. Hence the fluctuations of all reactive scalars can be related to that of a conserved scalar, usually mixture fraction in non-premixed combustion. Transport equations for the conditional averages are solved on a grid considerably coarser than that used for the LES. The required scalars for the LES code are then found by using their conditional average and a local mixture fraction PDF. In this paper we examine the ability of LES-CMC to predict localised transient extinction and re-ignition events within a turbulent non-premixed flame.

\section{Formulation}

The LES-CMC code developed in $[15,14]$ has been employed here. Filtering the governing equations for the flow yields equations for filtered mass, momentum and a conserved scalar, mixture fraction, $\xi$ which are to be solved. Details of the LES can be found elsewhere $[14,16]$, but here we note that the sub-grid scale stress tensor is modelled by the dynamic Smagorinsky model [17] and that a turbulent Schmidt number of 0.7 has been employed [18]. The sub-grid scale variance of the mixture fraction has been obtained by assuming a gradient type model $\widetilde{\xi^{\prime \prime}}=C_{V} \Delta^{2} \frac{\partial \widetilde{\xi}}{\partial x_{i}} \frac{\partial \widetilde{\xi}}{\partial x_{i}}$ where $C_{V}$ is a constant whose value is determined dynamically $[19,20]$. 
When the CMC model [21] is used, equations are solved for the conditionally filtered reactive scalars, the conditioning being done on $\xi$. The filtered value of the variable $f$ can then be obtained by integration over $\eta$-space $\widetilde{f}=\int_{0}^{1} \widetilde{f \mid \eta} \widetilde{\mathcal{P}}(\eta) d \eta[14]$. We assume that $\widetilde{\mathcal{P}}(\eta)$ has a $\beta$-function shape, which can be calculated based on the $\widetilde{\xi}$ and $\widetilde{\xi^{\prime \prime}}$.

The CMC equations can be derived by filtering the transport equations for the reactive scalars $Y_{\alpha}$ [11]. Using the primary closure assumption, the $\mathrm{CMC}$ equation becomes

$$
\frac{\partial Q_{\alpha}}{\partial t}+\widetilde{u_{i} \mid \eta} \frac{\partial Q_{\alpha}}{\partial x_{i}}=\widetilde{N \mid \eta} \frac{\partial^{2} Q_{\alpha}}{\partial \eta^{2}}+\widetilde{\omega_{\alpha} \mid \eta}+e_{f}
$$

where $Q_{\alpha}=\widetilde{Y_{\alpha} \mid \eta}$ is the conditionally filtered reactive scalar, $\widetilde{u_{i} \mid \eta}$ is the conditionally filtered velocity, $\widetilde{N \mid \eta}$ is the conditionally filtered scalar dissipation rate, $\widetilde{\omega_{\alpha} \mid \eta}$ is the conditionally filtered reaction rate, while the term

$$
e_{f}=-\frac{1}{\bar{\rho} \widetilde{\mathcal{P}}(\eta)} \frac{\partial}{\partial x_{i}}\left[\bar{\rho} \widetilde{\mathcal{P}}(\eta)\left(\widetilde{u_{i} Y_{\alpha} \mid \eta}-\widetilde{u_{i} \mid \eta} Q_{\alpha}\right)\right]
$$

is the sub-grid scale conditional flux and accounts for the conditional transport in physical space. All the species are assumed to have equal diffusivities and the Lewis number is assumed to be unity. A gradient model is used for the sub-grid scale conditional flux: $\widetilde{u_{i} Y_{\alpha} \mid \eta}-\widetilde{u_{i} \mid \eta} Q_{\alpha}=-D_{t} \frac{\partial Q_{\alpha}}{\partial x_{i}}[11]$. This model has given reasonable results in problems with significant spatial gradients of the conditional averages in LES of ignition [14]. Equation 1 without spatial transport terms and with a prescribed $\widetilde{N \mid \eta}$ has also been solved to give reference "laminar flamelet" solutions, denoted as "OD-CMC", and for initialization.

The terms $\widetilde{u_{i} \mid \eta}, \widetilde{N \mid \eta}$ and $e_{f}$ are unclosed and require modelling. The simple assumption that the conditional velocity is equal to the unconditional is made here, $\widetilde{u_{i} \mid \eta}=\widetilde{u_{i}}$, it is far from clear what model should be used here but this has the advantage of simplicity and it has been used in [14]. The Amplitude Mapping Closure (AMC) model is used to model the conditional scalar dissipation rate [22]. This model requires an unconditional filtered scalar dissipation rate $\widetilde{N}$ which is found by summing the resolved and subgrid contributions where the resolved component is

$$
\widetilde{N}_{\text {res }}=D\left(\frac{\partial \widetilde{\xi}}{\partial x_{i}}\right)^{2}
$$


and the sub-grid component is found by assuming that a mixing timescale is proportional to a velocity timescale [23] for the sub-grid $\tau_{t}=\Delta^{2} / \nu_{t}$ :

$$
\widetilde{N}_{s g s}=\frac{1}{2} C_{N} \frac{\nu_{t}}{\Delta^{2}} \widetilde{\xi^{\prime \prime 2}}
$$

The constant $C_{N}$ has been adjusted here to give a good match with the conditional scalar dissipation rate experimental data available for Flame D (discussed in Section 3.1).

Equation (1) is solved on a coarser grid than the LES and hence the information for $\widetilde{u_{i} \mid \eta}$ and $\widetilde{N \mid \eta}$ needed in the CMC must be averaged from the LES. This is done [15] by associating each LES cell with its closest CMC grid point, the required data for each CMC node is then mass weighted averaged over all LES cells associated with that node. In turn each LES cell uses the conditional averages, $Q_{\alpha}$, from its associated CMC node to find its new value of temperature and density. The conditional scalar dissipation rate is found by calculating the mass averaged $\widetilde{N}$ and $\mathcal{P}(\eta)$ for the whole CMC cell and applying the AMC model at the CMC level [15]. The LES equations are solved by the in-house Rolls-Royce code PRECISE, which is a finite-volume, low-Mach number code with block-structured mesh [16]. The CMC model has been implemented in PRECISE with coupling achieved through density and temperature. Details of of the implementation can be found in [14]. The only change here is that convection is discretised using a TVD scheme [24].

The Sandia piloted jet diffusion flames $\mathrm{D}\left(U_{j}=50 \mathrm{~m} / \mathrm{s}\right)$ and $\mathrm{F}\left(U_{j}=\right.$ $100 \mathrm{~m} / \mathrm{s}$ ) have been modelled [6]. Flame D has minimal local extinction whereas $\mathrm{F}$ has a large amount of local extinction and is close to global extinction. The jet has a diameter of $7.2 \mathrm{~mm}$ and contains $25 \%$ methane $-75 \%$ air by volume. This is surrounded by a pilot of outer diameter $18.2 \mathrm{~mm}$. The LES grid extended $80 \mathrm{D}$ in the axial and 20D in the radial directions. The grid is an 'O-ring' mesh using a minumum spacing of $0.25 \mathrm{~mm}$ across the pilot with spacing expanding smoothly downstream so that a total of approximately 1.3M nodes are used. The LES boundary conditions are the experimental velocity profiles across the jet and pilot [25]. No fluctuations are added to the inlet velocity but previous studies [11] have not found this to have a large effect as most of the turbulence in the jet is created in the shear layer. The mixture fraction is set to 1.0 in the jet, 0.0 in the co-flow and 0.27 in the pilot. This value is the mixture fraction that gives the pilot temperature $(1880 \mathrm{~K})$ and composition in a calculation of a low scalar dissipation rate laminar diffusion flame. 
First order closure is provided for the chemical reaction rate: $\widetilde{\omega_{\alpha} \mid \eta}=$ $\omega_{\alpha}\left(Q_{1}, Q_{2}, \ldots, Q_{n}\right)$, where $n$ is the number of reacting scalars. The ARM2 chemistry has been used that contains 19 species plus temperature [26]. This mechanism has previously been found to give good accuracy without a prohibitive computational cost [9]. The CMC grid has 51 nodes in $\eta$-space and $23 \times 23 \times 23$ nodes in an orthogonal mesh in physical space. The grid in the axial direction has an initial spacing of $3 \mathrm{~mm}$ until 3 jet diameters and then expands with a successive ratio of 1.3. The comparatively high number of nodes in the cross stream directions allows us to resolve the pilot and jet assembly with $9 \times 9$ of the nodes covering this region. This cross stream $\mathrm{CMC}$ resolution may also lessen the importance of the choice of model for conditional velocity. The boundary conditions for CMC are inert (291K at all $\xi$ values) in the jet and co-flow and a burning flamelet that gives the correct temperature at $\xi=0.27$ in the pilot. Figure 5 gives a schematic of the CMC grid and boundary conditions. The timestep was $\Delta t=2 \times 10^{-6} \mathrm{~s}$. The simulations were carried out on $323.0 \mathrm{GHz}$ dual core processors each with $2 \mathrm{~GB}$ of RAM per core. $7.2 \mathrm{~ms}$ of simulated time could be produced in 24 hours.

\section{Results and Discussion}

\subsection{Flame D}

As mentioned in Section 2 the constant used in finding unconditional sub-grid scalar dissipation rate in Eq. (4) was adjusted to give a good match between experimental results [27] and simulation for $\widetilde{N \mid \eta}$ in flame $\mathrm{D}$. The experimental data is available at three axial positions. To find a representative conditional average for a particular axial location, all the conditional averages at each CMC node at that axial position were averaged using PDF-weighted averaging, such that:

$$
\widetilde{\phi \mid}^{*}=\frac{\int \widetilde{\phi \mid \eta \widetilde{\mathcal{P}(\eta)} \widetilde{\rho \mid \eta} d V}}{\int \widetilde{\mathcal{P}(\eta) \rho \mid \eta} d V}
$$

where the integration is over all $\mathrm{CMC}$ nodes in all the points in time that are to be averaged. Results for $\widetilde{N \mid \eta}$ in Flame D are shown in Fig. 1. The CMC data is taken from the nearest axial node position to the point of interest, i.e. the CMC nodes that the LES cells at that point use. The averaging was performed on data taken every $0.2 \mathrm{~ms}$ of simulated time for a total of 
20ms. The correct trend is predicted and by tuning $C_{N}$ in Eq. (4) to a value of 42 reasonable agreement is found at the three axial locations. This value is within the range suggested by DNS [23]. It should be noted that this constant was the only parameter tuned to find these results and that to get better agreement at all three locations simultaneously would have required other modelling choices.

Using the scalar dissipation rate as found above it was found that the LES-CMC code predicted virtually no extinction anywhere for flame D. Conditionally averaged temperature at 7.5 jet diameters downstream is shown in Fig. 2. The experimental conditional averages are performed on data from across the jet and the CMC data is averaged across the jet and over time using Eq. (5). The slight overprediction by the CMC may be due to the absense of radiation in the model. This level of agreement and little extinction is seen at all axial positions for $\mathrm{D}$, but are omitted here for reasons of space. The unconditional data is also averaged using data from every $0.2 \mathrm{~ms}$ for 20ms. The centre-line mean and rms of mixture fraction for Flame D (Fig. 3(a)) are in very good agreement with experiment, indicating that the LES is predicting the correct mixing field. The good agreement for mixture fracture and conditional temperature leads to the excellent agreement for unconditional temperature seen in Fig 3(c). We conclude that the LES-CMC set-up used here is accurate for a flame far from extinction. For Flame F the agreement is again very good although the agreement is not as good for temperature (Fig. 3(d)), particularly for rms. This is most likely due to the incorrect degree of extinction predicted in parts of the flow, which is discussed below.

\subsection{Flame $F$}

The LES-CMC code with settings equal to those used for Flame D was applied to simulate Flame F, which shows a large amount of local extinction. All averages for $\mathrm{F}$ were found using data from every $0.2 \mathrm{~ms}$ for $16 \mathrm{~ms}$. Figure 4 shows instantaneous contours of resolved temperature for both flames D and F. While the contour for D shows an unbroken region of high temperature extending to the pilot, that for F shows patches of low temperature (1200K) corresponding to localised extinction. This can be seen more clearly in Fig. 5 which shows a close up of $\mathrm{OH}$ mass fraction contours at the same instant as the temperature contour in Fig. 4. The $\mathrm{OH}$ contour also reveals that the location of the extinctions correspond to the CMC grid nodes that have extinguished in mixture fraction space. Evidence of this CMC extinction 
can be clearly seen in Fig. 6, which shows conditional temperatures from several time instants for $\mathrm{CMC}$ nodes with a radial position between $5 \mathrm{~mm}$ and $7 \mathrm{~mm}$ at $7.5 \mathrm{D}$ axial position. The conditional distributions at this location cover a wide range from fully burning to extinguished. This range is very close to that seen for the scatter plot of experimental data seen at the same location. Plotted on the same figure is the experimental conditional average and the PDF weighted average of the $Q_{T}$ produced according to Eq. (5). We see here how the LES-CMC produces a reduced time averaged conditional temperature (with a peak of $1400 \mathrm{~K}$ ) from $Q_{T}$ that fluctuates widely in time between extintion and re-iginition.

Selected time series for $N \widetilde{\mid \eta=0.5}, \quad \widetilde{T \mid \eta=\xi_{s t}}$ and $\widetilde{O H \mid \eta}=\xi_{\text {st }}$ are shown in Fig. 7 for three CMC nodes at a radial position of $6.75 \mathrm{~mm}$. Also marked on the scalar dissipation plot is the value that would be required to extinguish the flamelet by itself with no tranport terms; this is labelled '0D extinction.' Note that due to the use of the AMC model the ratio of $N \mid \eta=0.5$ to $N \mid \eta=\xi_{s t}$ is constant. At $y / D=5$ the scalar dissipation rate several times exceeds the steady flamelet extinction value and a response can be seen in the $O H$ trace which decreases. Some of these events do not last long enough for a full extinction to take place. Others, however, do result in zero $\mathrm{OH}$ and temperatures down to 1300-1400K (other events must result in lower temperatures; see the spread in the $Q_{T}$ in Fig. 6). Note that the first peak in scalar dissipation rate which does cause an extinction occurs when $O H$ is already reduced. At $y / D=6.6$ the $0 \mathrm{D}$ extinction dissipation rate is only exceeded briefly however two clear extinction events are observed, the second lasting more than $1 \mathrm{~ms}$. Hence it can be deduced that these extinctions must be caused in part by transport from neighbouring CMC cells. Re-ignition of flamelets for which $O H$ has been completely reduced must also be due to transport from neighbouring burning flamelets, since relaxation of the $N \widehat{\mid \eta=0.5}$ will not result by itself in ignition (as revealed by numerical experiments with 0D-CMC using the local three-dimensional LES-CMC as initial condition for $Q_{T}$ and $Q_{\alpha}$ ).

Time and cross-stream averaged $Q_{T}$ and mass fraction of $\mathrm{O}_{2}$ and $\mathrm{OH}$ are shown in Fig. 8 together with the corresponding experimental data. As was seen in Fig. 6 the correct amount of extinction is seen at 7.5 jet diameters and very good agreement is seen for temperature and $\mathrm{O}_{2}$. Note that, for both experiment and simulation, the $\mathrm{OH}$ mass fraction at this point takes intermediate values that could not be sustained in a steady flamelet, 
showing the averaged effect of many transient extinctions. By 15 diameters downstream the amount of extinction is underpredicted and there are several possible reasons for this. As mentioned earlier many of the modelling choices used in LES-CMC remain to be decided and these may have an effect. It is possible that numerical diffusion in the current CMC transport scheme does not allow exinctions to be advected downstream as they should. A final point is that, while the mean scalar dissipation rate at 15 diameters matches experimental data in Fig. 1, correctly matching the fluctuations of this quantity will also be necessary to correctly predict extinctions and we do not know if this is the case. By 30 jet diameters there is only a small amount of extinction evident in the experimental results and the accuracy of the simulation improves.

An example of the importance of CMC grid resolution in the cross stream direction is shown in Fig. 9 in which conditional temperature is shown at two radial positions $2 \mathrm{~mm}$ apart at the same axial position, $y / D=7.5$. A lower average conditional temperature is observed at $6 \mathrm{~mm}$ than at $8 \mathrm{~mm}$, indicating that more extinction is observed closer to the centre of the jet. This trend is correctly predicted by 3D CMC. Finally, the unconditional averages are shown in Fig. 10. These radial profiles of mixture fraction and temperature have been found by averaging resolved LES data both in time and azimuthally at a given radius. We see that mean and rms of mixture fraction is well predicted. The resolved temperature profiles are reasonable and follow our expectations from the conditional results presented above in Fig. 8. At $y / D=7.5$ the reduced peak temperature due to localised extinction is correctly predicted, whereas temperature is overpredicted at $y / D=15$ due to not enough extinction being predicted at this location. By $y / D=30$ the prediction is again good as $\mathrm{CMC}$ and experiment return to a low extinction state.

The present results suggest that the LES-CMC formulation, with a refined multi-dimensional CMC grid, can capture localised extinction due to excursions of the scalar dissipation above the extinction value and can also capture re-ignition due to heat and species flux from neighbouring fluid. The accuracy of the predictions is sensitive to the conditional scalar dissipation model, the model used for $e_{f}$, and to the numerical diffusion associated with the CMC physical transport discretization schemes. 


\section{Conclusions}

An LES-CMC code has been applied to the Sandia piloted jet flames D and F. A 3D CMC grid was used with enough resolution in the cross stream directions to resolve the pilot and jet assembly. The constant used to find sub-grid scale unconditional scalar dissipation rate was adjusted in order to give a good match for the results of flame D. The same settings were then applied to flame F. CMC nodes within the flame underwent a succession of transient extinction and re-ignition events. These events were observed to be the result of fluctuating conditional scalar dissipation rate and transport from neighbouring cells in the 3D grid. These extinctions in the CMC grid lead to low resolved temperatures in the LES simulation of the flame and zero $\mathrm{OH}$ mass fraction. Good agreement was found with experiment for conditional and unconditional average temperature at $y / D=7.5$, where the correct amount of extinction was observed. The extent of localised extinction was underpredicted as not enough extinction was observed at 15 jet diameters. It should be noted that the only parameter adjusted during this work was the constant used for $\widetilde{N}_{s g s}$ in Eq. (4) there are several other options that could be tried such as how conditional scalar dissipation rate is found from the unconditional [15]. These and steps to reduce numerical diffusion in the CMC grid could change the area over which local extinction is observed.

\section{Acknowledgements}

This work has been funded by the European Commission (Project "TECCAE"). Thanks to Dr. A. Triantafyllidis for assistance with the LES-CMC code.

[1] H. Pitsch, Ann. Rev. Fluid Mech. 38 (2006) 453-482.

[2] F. Di Mare, W. P. Jones, K. R. Menzies, Combust. Flame 137 (2004) 278-294.

[3] H. Pitsch, H. Steiner, Phys. Fluids 12 (2000) 2541-2554.

[4] C. D. Pierce, P. Moin, J. Fluid Mech. 504 (2004) 73-97.

[5] R. Mustata, L. Valiño, C. Jiménez, W. P. Jones, S. Bondi, Combust. Flame 145 (2006) 88-104. 
[6] R. S. Barlow, J. Frank, Proc. Combust. Inst. 27 (1998) 1087-1095.

[7] R. P. Lindstedt, S. A. Louloudi, E. M. . Váos, Proc. Combust. Inst. 28 (2000) 149-156.

[8] J. Xu, S. B. Pope, Combust. Flame 123 (2000) 281-307.

[9] R. R. Cao, S. B. Pope, Combust. Flame 143 (2005) 450-470.

[10] M. Ihme, H. Pitsch, Combust. Flame 155 (2008) 90-107.

[11] S. Navarro-Martinez, A. Kronenburg, F. Di Mare, Flow Turb. Combust. 75 (2005) 245-274.

[12] S. Navarro-Martinez, A. Kronenburg, Proc. Combust. Inst. 31 (2007) 1721-1728.

[13] S. Navarro-Martinez, A. Kronenburg, Proc. Combust. Inst. 32 (2009) 1509-1516.

[14] A. Triantafyllidis, E. Mastorakos, R. Eggels, Combust. Flame 156 (2009) 2328-2345.

[15] A. Triantafyllidis, E. Mastorakos, Flow Turb. Combust. 84 (2010) 481512.

[16] S. James, J. Zhu, M. S. Anand, AIAA J. 44 (2006) 674-686.

[17] M. Germano, U. Piomelli, P. Moin, W. Cabot, Phys. Fluids 3 (1991) 1760-1765.

[18] N. Branley, W. P. Jones, Combust. Flame 127 (2001) 1914-1934.

[19] A. W. Cook, J. J. Riley, Phys. Fluids 6 (1994) 2868-2870.

[20] C. D. Pierce, P. Moin, Phys. Fluids 10 (1998) 3041-3044.

[21] A. Y. Klimenko, R. W. Bilger, Prog. Energy Combust. Sci. 25 (1999) 595-687.

[22] E. O’Brien, T.-L. Jiang, Phys. Fluids 3 (1991) 3121-3123.

[23] C. Jiménez, F. Ducros, B. Cuenot, B. Bédat, Phys. Fluids 13 (2001) 1748-1754. 
[24] T. J. Chung, Computational fluid dynamics, Cambridge University Press, Cambridge, 2002.

[25] C. Schneider, A. Dreizler, J. Janicka, E. Hassel, Combust. Flame 135 (2003) 185-190.

[26] C. J. Sung, C. K. Law, J. Y. Chen, Proc. Combust. Inst. 27 (1998) 295-304.

[27] A. N. Karpetis, R. S. Barlow, Proc. Combust. Inst. 30 (2005) 665-672. 


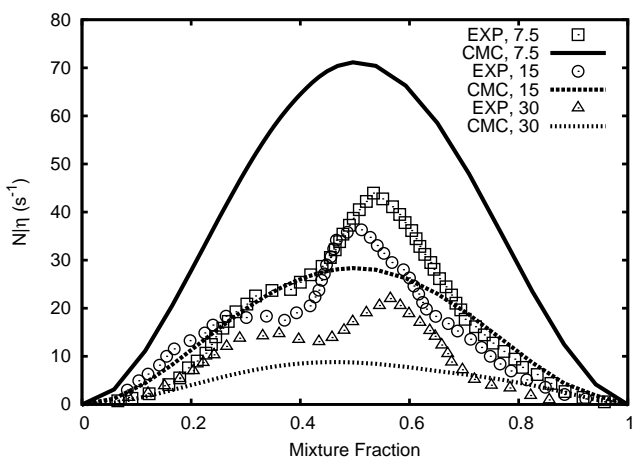

Figure 1: Conditional Scalar dissipation rate at three axial locations (indicated $y / D$ ) for flame D. Experimental data [27] and averaged CMC data found from Eq. (1).

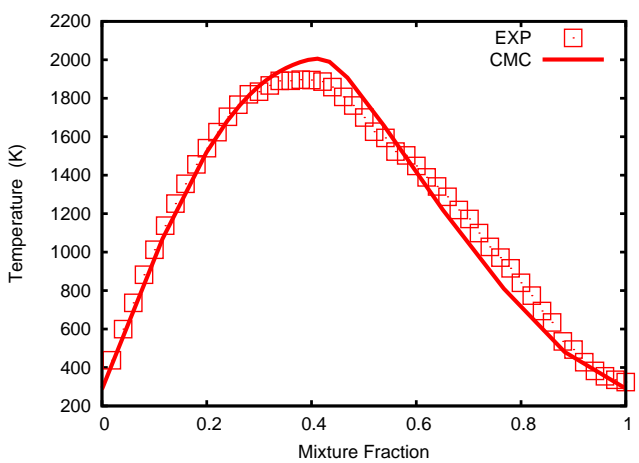

Figure 2: Conditional average temperature for Flame D at 7.5 jet diameters. Experimental data from [6]. 

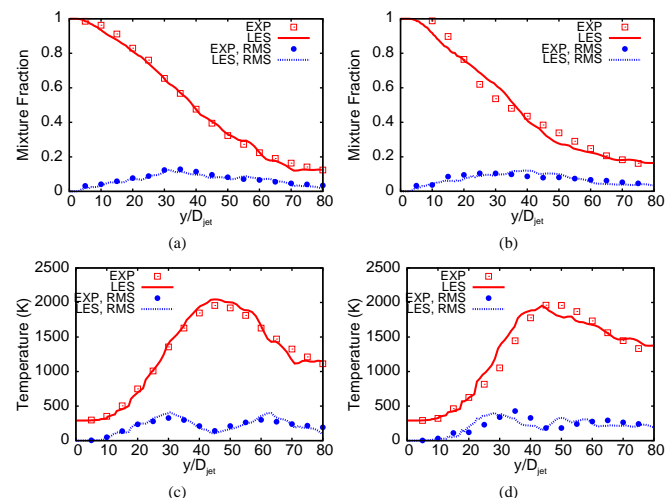

Figure 3: Average resolved mixture fraction (top) and temperature (bottom) along centreline for Flames D (left) \& F (right). Experimental data from [6].

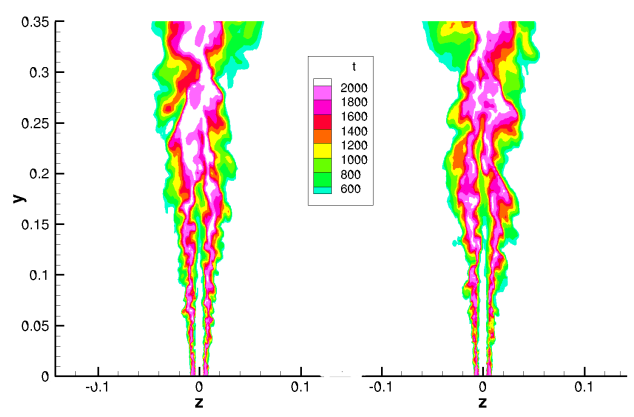

Figure 4: Instantaneous contour of resolved temperature (in K). On the left Flame D and on the right flame $\mathrm{F}$. Axes in $\mathrm{m}$. 


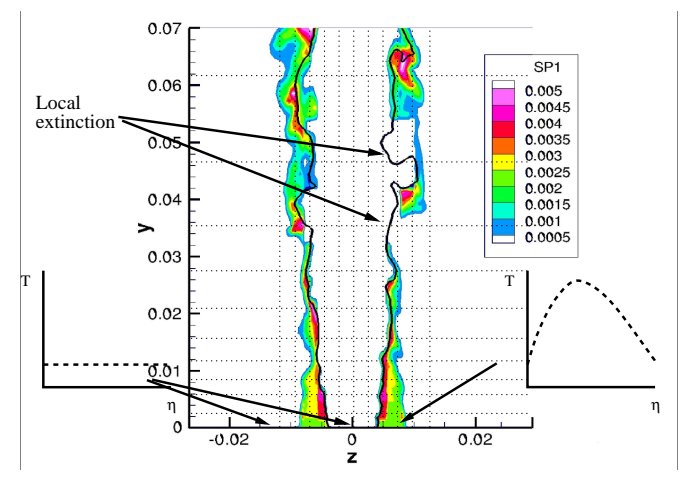

Figure 5: Instantaneous contour of $\mathrm{OH}$ mass fraction with $\xi_{s t}$ contour (black line) for Flame F. The early part of the CMC grid and the CMC boundary conditions are included for reference. Axes in $\mathrm{m}$.

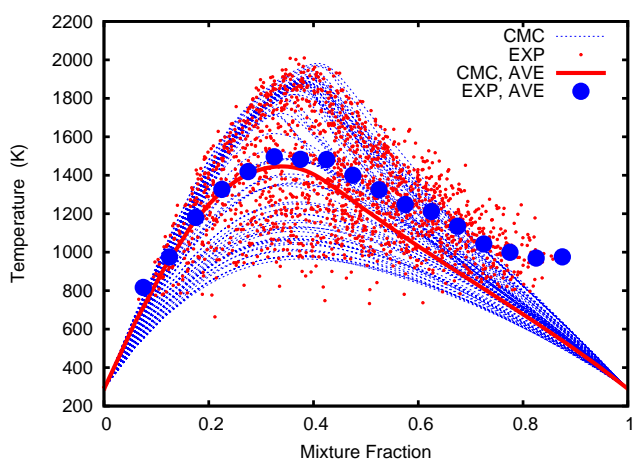

Figure 6: Conditonal temperature at $y / D=7.5$ and $r=6 \mathrm{~mm}$ for Flame F. Scatter data and conditional average from experiment [6]. Instantaneous $Q_{T}$ ('CMC') and PDFweighted average $Q_{T}$ ('CMC, AVE.'). 

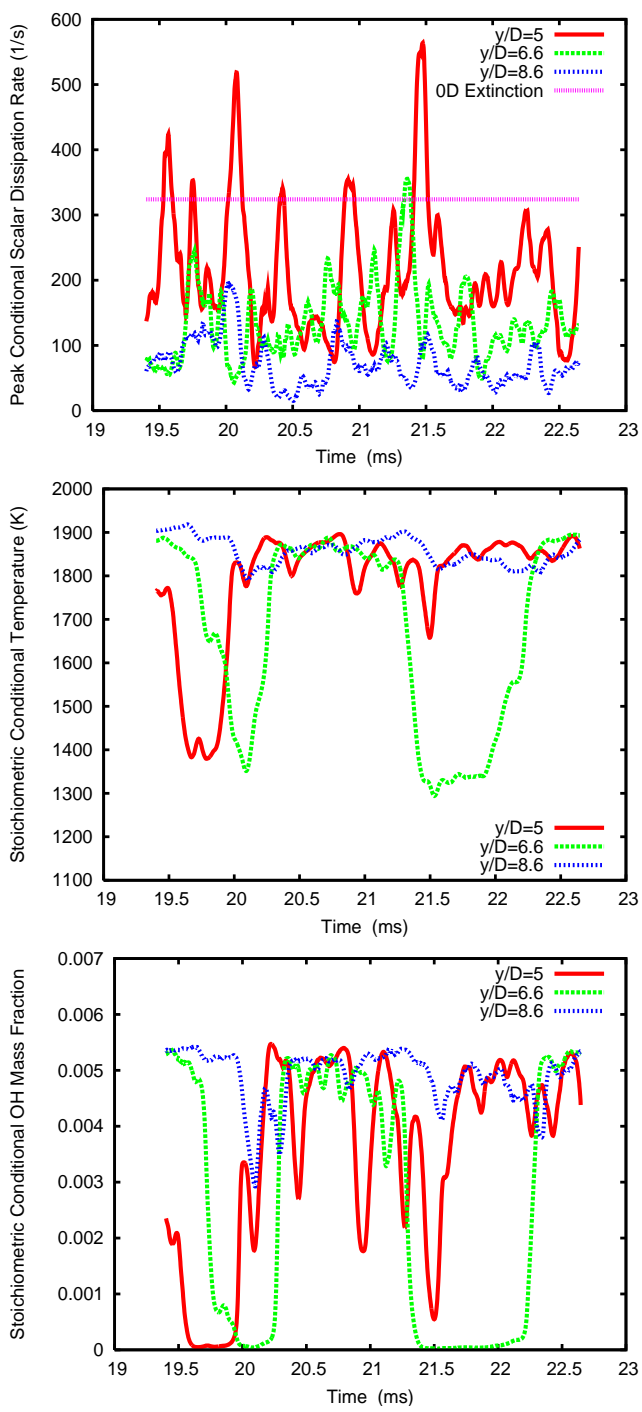

Figure 7: Time series of peak conditional scalar dissipation rate (top) and stoichiometric conditional temperature (middle) and $O H$ mass fraction (bottom) for three axiallyadjacent $\mathrm{CMC}$ nodes at a radial position of $6.75 \mathrm{~mm}$ for Flame F. 

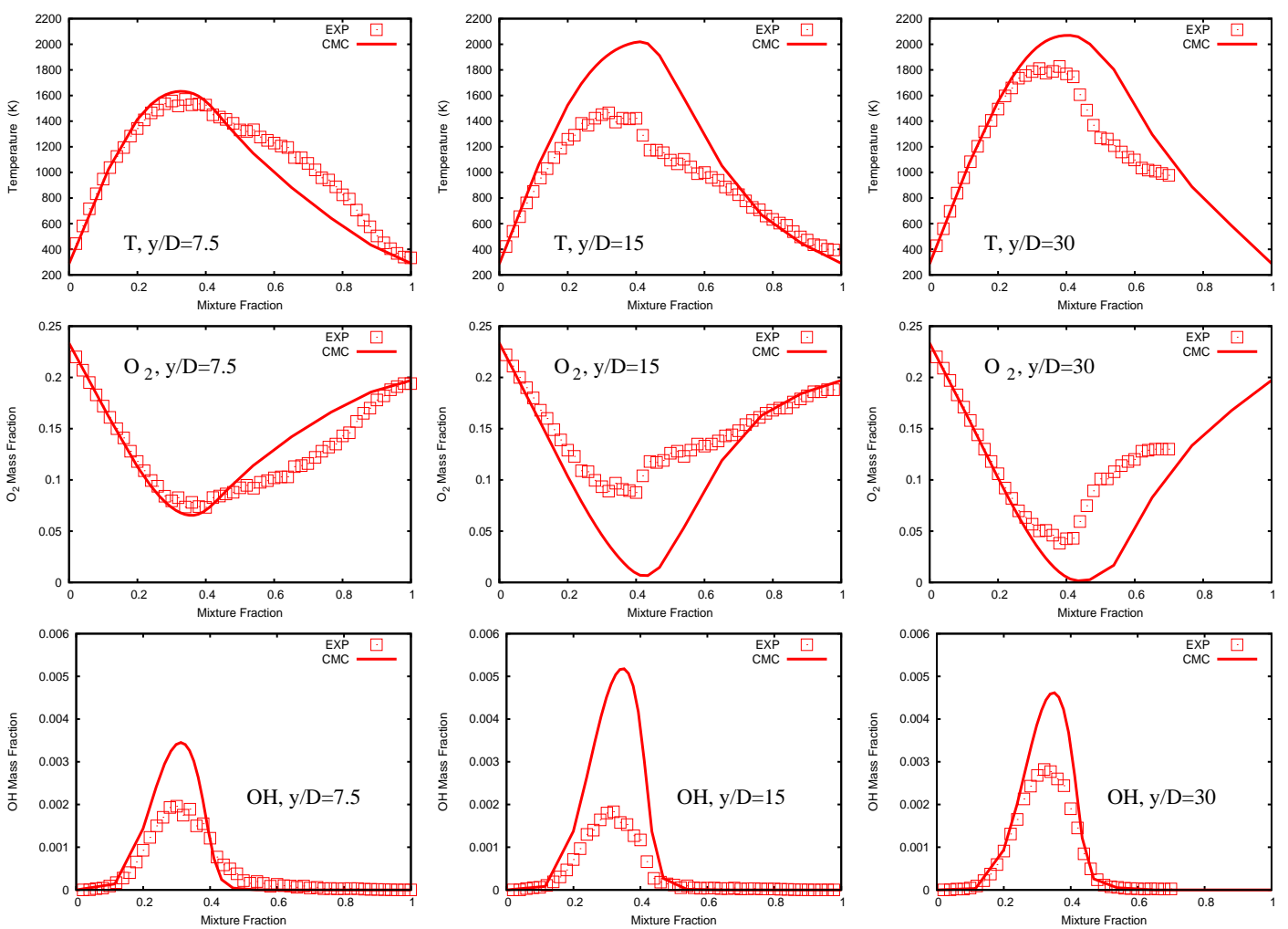

Figure 8: Conditional averages of temperature, $\mathrm{O}_{2}$ and $\mathrm{OH}$ mass fraction for Flame F. Experimental results from [6].

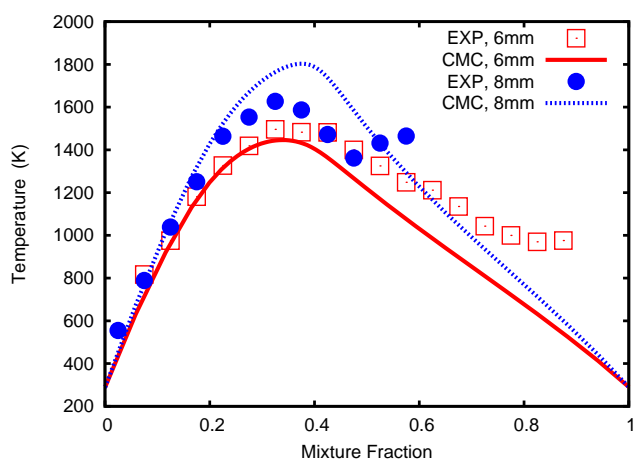

Figure 9: Conditonal average temperature at $\mathrm{y} / \mathrm{D}=7.5$ and radial positions of $6 \mathrm{~mm}$ and $8 \mathrm{~mm}$ for Flame F. CMC results produced by PDF - weighted averaging. Experimental results from $[6]$. 

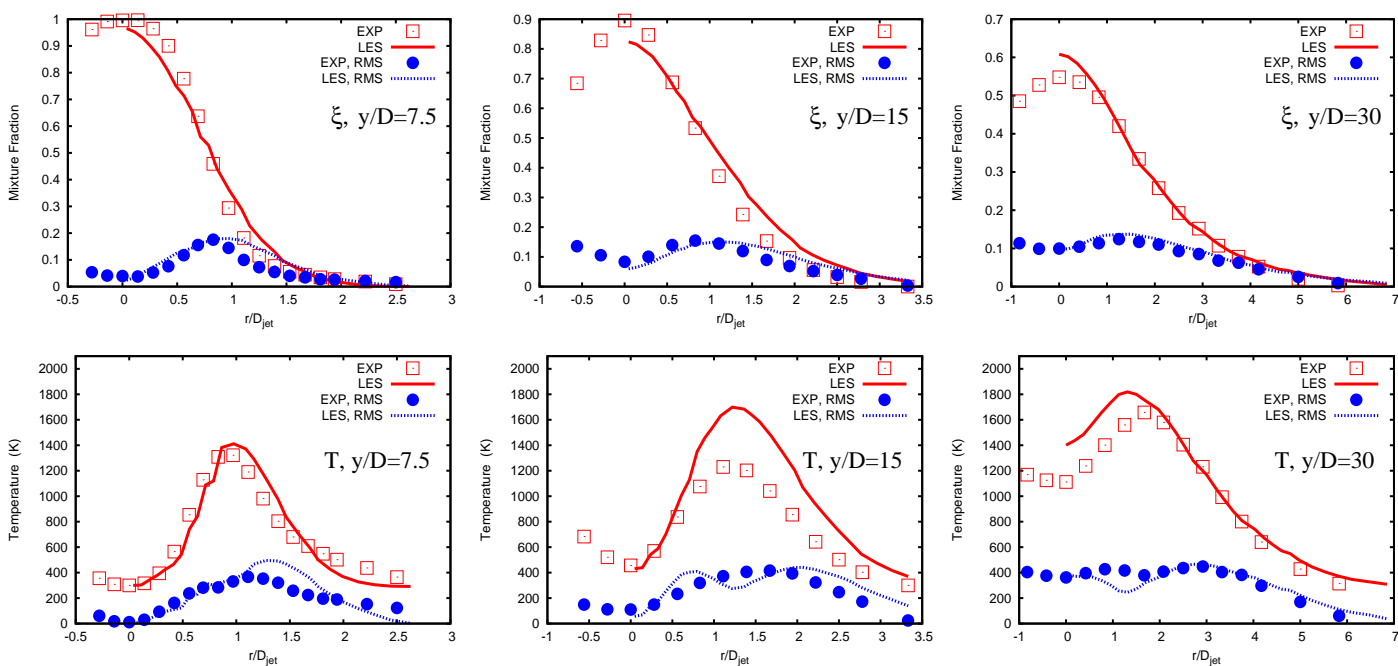

Figure 10: Radial profiles of mean and rms of resolved mixture fraction and temperature for Flame F. Experimental results from [6]. 